\title{
The Stickler syndrome presenting as a dominantly inherited cleft palate and blindness*
}

\author{
JUDITH G. HALL and HENRY HERROD \\ From the University of Washington Medical School, Division of Medical Genetics, and Children's \\ Orthopedic Hospital and Medical Center, Seattle, Washington, USA \\ Summary. The Stickler syndrome is a newly recognized, but probably rela- \\ tively frequent inherited generalized connective tissue disorder involving skeleton, \\ eye, and oro-facial structures. A family with three affected generations is dis- \\ cussed. Severe myopia leading to blindness, cleft palate, or submucous cleft, Pierre \\ Robin anomaly, premature degenerative arthritis, or a family history of any of these \\ indicates further evaluation.
}

The Stickler syndrome is a newly recognized, but probably relatively frequent inherited connective tissue disorder involving skeleton, eye, and orofacial structures. We have recently seen a family which presented with dominantly inherited cleft palate and blindness.

\section{Case report}

A 4-year-old Caucasian girl was referred because of recurrent otitis media, severe myopia ( -17.5 diopters) relative hypotonia, and anaemia. She had a cleft palate which had been repaired at 1 year of age and mild midfacial hypoplasia. She did not have arthritis, hyperextensibility of joints or, arachnodactyly. Her family history was remarkable (Fig. 1) in that she had an older brother (V.1) who died unexpectedly at $3 \frac{1}{2}$ months of age and had had the Pierre Robin anomaly (micrognathia, cleft palate, and glossoptosis) treated with tracheostomy.

Her 6-year-old sister (V.2) had cleft palate with a normal sized jaw, flattening of the right face which had decreased with age, mild mid-facial hypoplasia, severe myopia ( -20.0 and -23.0 diopters), and strabismus (Fig. 2). She had no arthritis, but radiographs showed the changes seen in Fig. 3.

The 34-year-old mother (IV.3) had also had Pierre Robin anomaly, facial asymmetry (with a flattened left face), myopia with cataract and retinal detachment in the right eye, and degenerative uveitis, retinal detachment

Received 12 July 1974.

* This study was supported in part by the National Institute of Health Grant No. 5 P01 GM 15253-07 and Grant No. 5501 RR 0565505 and the National Foundation-March of Dimes Grant CA-90.

Reprint requests to Judith G. Hall MD, Division of Medical Genetics, Children's Orthopedic Hospital, 4800 Sand Point Way, N.E., Seattle, Washington 98105, USA. and glaucoma in the left eye, post-operative ptosis of the left eyelid, and mild arthritis of her arms and fingers during the last few years (Fig. 2).

The 31-year-old uncle (IV.4) had bilateral cataracts diagnosed at $1 \frac{1}{2}$ years of age. Removal of the left cataract led to retinal detachment, glaucoma, and enucleation. The right eye had retinal degeneration in addition to the cataract and eventually developed glaucoma and was enucleated. He also had facial asymmetry and postoperative ptosis. He has a submucous cleft of the posterior quarter of his palate and was late in learning to

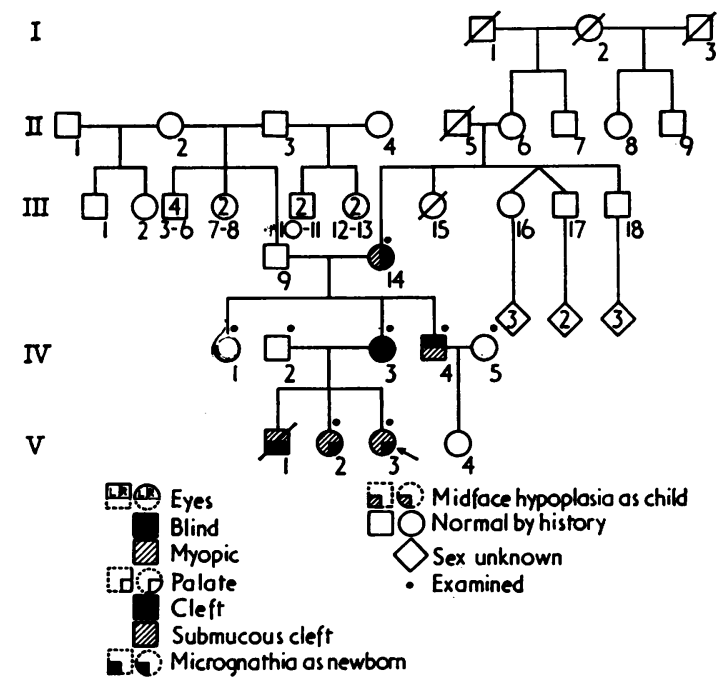

Fig. 1. Pedigree. 


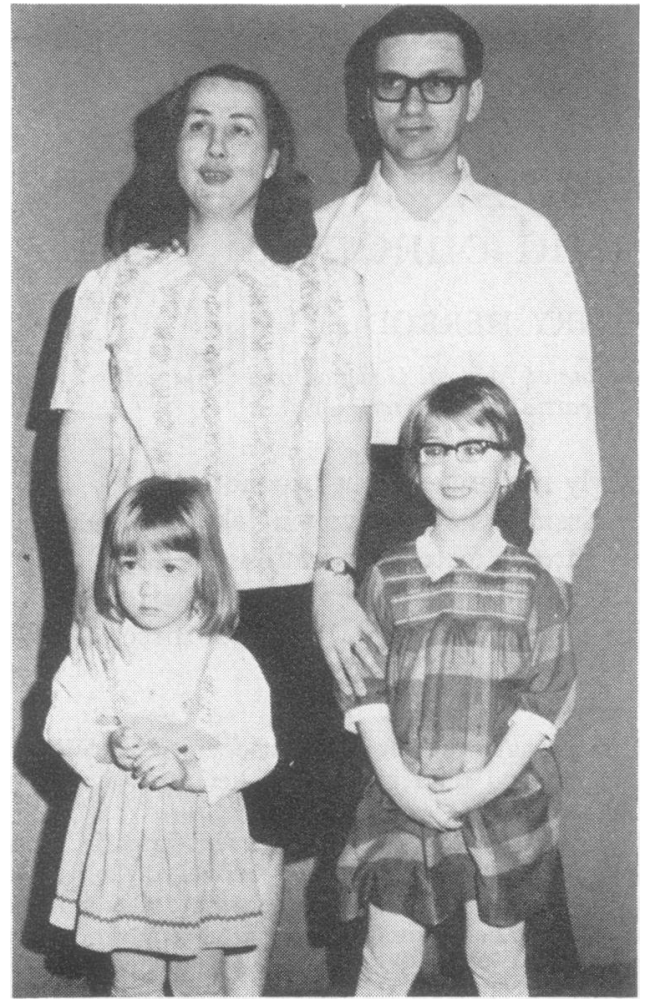

Fig. 2. IV.3 with normal husband and affected daughters, V.3 and V.2. Note mild facial asymmetry, ptosis, normal jaw size and full mid face in IV.3. Note mild facial asymmetry, thick glasses, strabismus and round face in V.2. Note mid-facial hypoplasia and round face in V.3.

speak and walk, but was of normal intelligence as an adult.

The grandmother (III.14) was born when her father was 46 years of age and her mother 25 . She had cleft palate, but normal mandible and a symmetric face. She had a cataract and glaucoma in the left eye leading to blindness and enucleation. She has -11.75 diopter myopia in her right eye. She has had arthritis of the hips, hand, and shoulders over the last few years.

There are no other family members with cleft palate, myopia, or arthritis. No consanguinity is known. None of the affected family members have arachnodactyly, hyperextensible joints, nerve deafness, scoliosis or mental retardation. Blood group linkage studies were non-informative.

\section{Discussion}

In 1965, Stickler et al first described a family with an autosomal dominant disorder of connective tis- sue which they called 'hereditary progressive arthroopthalmopathy' (Stickler et al, 1965; 1967). These patients had severe degenerative joint changes and progressive myopia with spontaneous retinal detachment leading to blindness. Since that time, numerous other cases have been described or have been recognized as having been reported under a different name and it has become clear that within a given family, great variability in severity and in organ system involvement can occur. The clinical features of the Stickler syndrome fall into three major categories. (1) Skeletal: degenerative arthritis, mild spondylo-epiphyseal dysplasia, large joints, Marfanoid habitus, and hyperextensibility. (2) Ocular: myopia, retinal detachment, retinal degeneration, glaucoma, cataract, and blindness. (3) Oro-facial: cleft palate, Pierre Robin anomaly, submucous cleft, and hypoplasia of the midface. Herrmann et al (1975) have suggested that the Stickler syndrome may be the most common autosomal dominant connective tissue disorder in the north American Midwest.

Abnormal joint manifestations seem to be the most common, although not necessarily the most dramatic feature of the Stickler syndrome (Schreiner et al, 1973; Hermann et al, 1975). Bone and cartilage abnormalities are reflected in the $x$-rays as a mild spondylo-epiphyseal dysplasia and apparently predisposed to premature degenerative changes and then arthritis. Arthritic symptoms usually do not occur until late childhood or adolescence and may not develop until middle age or later. The radiological changes (Spranger, 1968; Schreiner et al, 1973) may be present from birth and are characterized by overtubulation of long bones, mild flattening and irregularity of epiphyses, mild irregularity and flattening of vertebrae, hypoplasia of the midfacial bones and steep base of the skull (Fig. 3). Schreiner et al (1973) pointed out that as in the present family, the radiological changes were often the most helpful clue to a specific diagnosis of the Stickler syndrome in otherwise asymptomatic individuals in an affected family.

The typical body habitus described by Stickler et al (1965) is slender with disproportionately long arms and legs, but definitely affected individuals have been short and stocky (Schreiner et al, 1973; Herrmann et al, 1975). Some patients have had prominent joints (which may be noted at birth [Stickler et al, 1965]), decreased musculature, hypotonia, scoliosis, increased lumbar lordosis, and hyperextensibility of joints (Herrmann et al, 1975). Among the members of the present family, none had arachnodactyly, hyperextensibile joints, nor incapacitating degenerative arthritis. Nevertheless, 

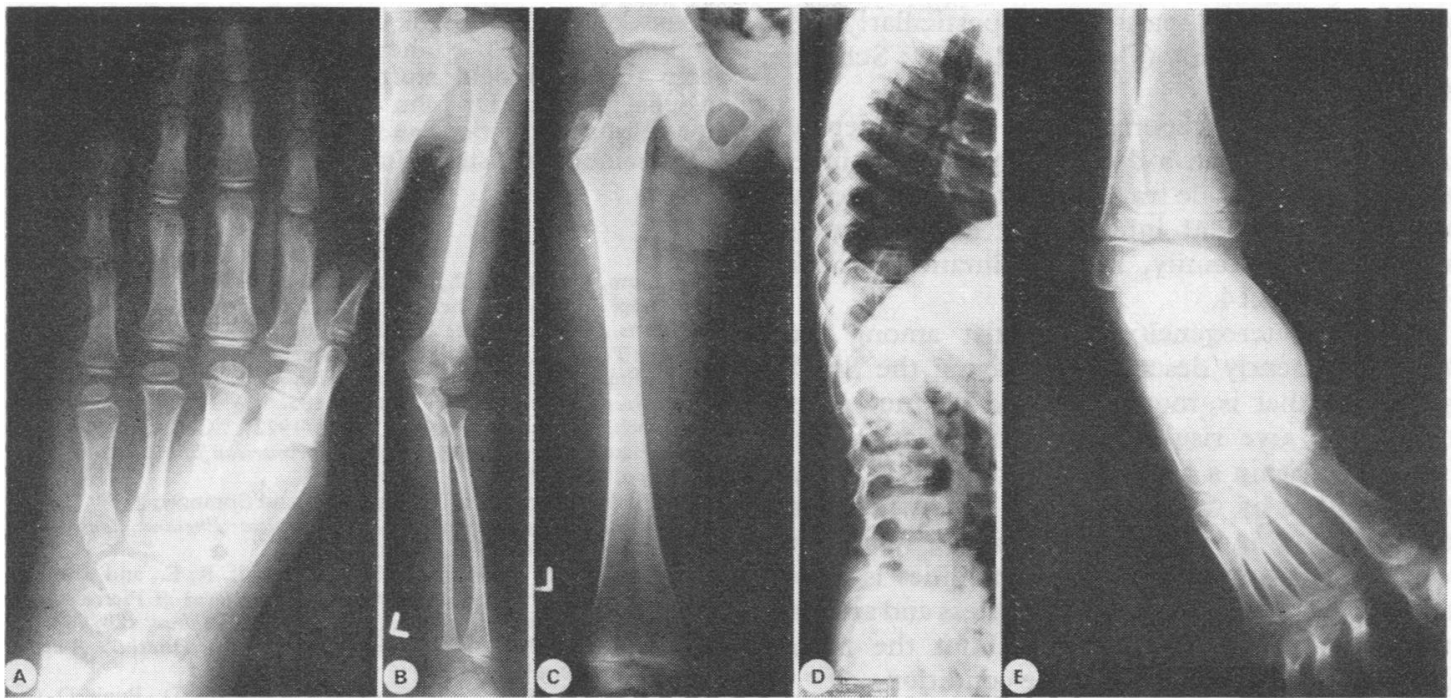

Fig. 3. V.2. A: $X$-ray of hand showing mild flattening of phalangeal epiphysis, irregularity and missing aspect of distal radial epiphysis, crowding of carpals and long phalanges. B: $X$-ray of arm showing relatively narrow shaft of ulna with broad metaphysis. C: $X$-ray of leg showing relatively narrow shaft and broad metaphyses of femur with coxa valga of femoral neck and broad capital femoral epiphyses. D: $X$-ray of spine. Note anterior wedging and mild irregularity of vertebral surfaces, particularly in the thoracic area. E: $X$-ray of ankle. Note irregularity of distal tibial epiphysis with flattening laterally.

$x$-ray films demonstrate definite involvement of the skeleton. Perhaps because of significant eye involvement, the members of this family had less strenuous physical activity and thereby saved their joints some of the usual wear and tear. Avoidance of strenuous activity involving the joints is probably indicated in this condition.

The variety and progressive nature of the eye anomalies seen in patients with the Stickler syndrome is well demonstrated by the present family. All of these patients had severe myopia. Herrmann et al (1975) estimate two-thirds of patients have myopia. Cataracts, non-traumatic retinal detachment leading to blindness, retinal degeneration, glaucoma, ptosis, and oesotropia have all been reported and were seen in this family. Lesions can be unilateral or bilateral. Potentially, careful opthalmologic observation, avoidance of trauma, patient education, and aggressive therapy could prevent some of the retinal detachments and complications of glaucoma (Spranger, 1968; Knobloch and Layer, 1972; Hirose et al, 1973).

The oro-facial features of the Stickler syndrome were not elaborated by Stickler et al $(1965 ; 1967)$, but appear to be a nearly constant feature. Midfacial hypoplasia, round facies, and facial asymmetry have been noted (Knobloch and Layer, 1972; Schreiner et al, 1973; Herrmann et al, 1975).
Micrognathia so severe as to be called Pierre Robin anomaly has been reported frequently (Knobloch and Layer, 1972; Opitz et al, 1972; Herrmann et al, 1975). The disproportion of facial structures appears to improve with age with growth of the midface and mandible such that asymmetry is less apparent by adulthood (Knobloch and Layer, 1972).

The palate in patients with the Stickler syndrome may be normal or highly arched, have a submucous cleft or a complete cleft of the hard palate. A bifid uvula may be the only finding. Between one-third and one-quarter of the reported cases of the Stickler syndrome have clefting of the palate but in some families, such as the one reported here, the number of family members with palatal involvement is much greater (Knobloch and Layer, 1972). Cleft lip has not been seen. Many patients present as Pierre Robin anomaly and consequently require complex management during the newborn period. As with all patients who have cleft palates, Stickler patients with cleft palates are prone to recurrent otitis and secondary conductive hearing loss. In addition, neurosensory hearing loss in patients with the Stickler syndrome has been described (Stickler and Pugh, 1967). The combination of ophthalmological and palate problems seen in the Stickler syndrome underlines the necessity for careful eye examination and ophthalmological follow up of any 
patient with cleft palate and particularly with Pierre Robin anomaly (Opitz et al, 1972; Schreiner et al, 1973).

Of the families reported with the Stickler syndrome, all have had a dominant pattern of inheritance. Male to male transmission has occurred and autosomal dominant inheritance can be assumed. In the present family, a new mutation probably occurred in III.14.

Genetic heterogeneity may exist among those families presently described as having the Stickler syndrome, that is, more than one distinct genetic lesion may give rise to a similar clinical picture. However, within a given family, marked variability of expression of the gene occurs both with regard to severity of involvement and organ systems involved. Recognition of affected families is important in order to try to prevent blindness and arthritis, and so that families can learn about the natural history and genetic nature of the condition.

The authors are grateful to Dr Roger Johnson, Dr Robert H. Johns, Dr Samuel H. Tarica, Jr, Dr J. I. Moreland, Dr Richard L. Voorhees, and Dr Gilbert Eade for making available additional historical information on the family; to Dr Eliose Giblett of the Seattle
King County Blood Bank for performing blood grouping and serum protein studies; to $\mathrm{Dr}$ Frederick Hecht for help during a field study; to Dr David Smith, Dr Michael Cohen, Dr John Opitz and Dr Arno Motulsky for helpful suggestions and criticism, and to Miss Robin McIlvaine for assistance in the preparation of the manuscript.

\section{REFBRENCES}

Herrmann, J., France, T., Spranger, J., and Opitz, J. (1975). The Stickler syndrome (heriditary arthroophthalmopathy). Birth Defects, Original Article Series, 11, no. 2, 77-103.

Hirose, T., Lees, K. Y., and Schepens, C. L. (1973). Wagner's hereditary vitreoretinal degeneration and retinal detachment. Archives of Ophthalmology, 89, 176-185.

Knobloch, W. E., and Layer, J. M. (1972). Clefting syndromes associated with retinal detachment. American fournal of Ophthalmology, 73, 517-530.

Opitz, J. M., France, T., Herrmann, J., and Spranger, J. W. (1972). The Stickler syndrome. (Letter.) New England fournal of Medicine, 286, 546-547.

Schreiner, R. L., McAlister, W. H., Marshall, R. E., and Shearer, W. T. (1973). Stickler syndrome in a pedigree of Pierre Robin syndrome. American fournal of Diseases of Children, 126, 86-90.

Spranger, J. (1968). Arthro-ophthalmopathia hereditaria. Annales de Radiologie, 11, 359-364.

Stickler, G. B., Belau, P. G., Farrell, F. J., Jones, J. D., Pugh, D. G., Steinberg, A. G., and Ward, L. E. (1965). Hereditary progressive arthro-ophthalmopathy. Proceedings of the Staff Meetings of the Mayo Clinic, 40, 433-455.

Stickler, G. B. and Pugh, D. G. (1967). Hereditary progressive arthro-ophthalmopathy. II. Additional observations on vertebral abnormalities, a hearing defect and a report of a similar case. Proceedings of the Staft Meetings of the Mayo Clinics, 42, 495-500. 